\title{
Coloração da gordura e qualidade da carne de ovelhas de descarte abatidas em distintos estágios fisiológicos
}

\author{
[Fat coloring and meat quality of culling ewes slaughtered at distinct \\ physiological stages] \\ R.S.B. Pinheiro ${ }^{1}$, A.M. Jorge ${ }^{2}$, H.B.A. Souza ${ }^{3}$, M.M. Boiago ${ }^{3}$ \\ ${ }^{1}$ Faculdade de Engenharia de Ilha Solteira - UNESP \\ 15385-000 - Ilha Solteira, SP \\ ${ }^{2}$ Faculdade de Medicina Veterinária e Zootecnia - UNESP - Botucatu, SP \\ ${ }^{3}$ Faculdade de Ciências Agrárias e Veterinárias - UNESP - Jaboticabal, SP

\section{RESUMO}

Avaliaram-se a qualidade física do músculo Longissimus lumborum de ovelhas de descarte da raça Santa Inês, abatidas em diferentes estágios fisiológicos, a cor da gordura subcutânea desse músculo e as possíveis correlações entre as variáveis de qualidade da carne. Utilizaram-se 21 ovelhas, distribuídas nos seguintes tratamentos: $\mathrm{T} 1=$ ovelhas que permaneceram por 60 dias em lactação com seus respectivos cordeiros e abatidas um dia após o desmame das crias; T2= ovelhas que permaneceram por 60 dias em lactação com seus respectivos cordeiros e mais um período aproximado de 30 dias sem os cordeiros e posteriormente abatidas; e T3= ovelhas que não pariram durante o ano. A cor da carne e da gordura subcutânea não foram influenciadas pelo estágios fisiológico dos animais no momento do abate, com exceção da luminosidade da carne (T1= 41,13; T2=37,56 e T3=38,12 L*). A qualidade física da carne apresentou pouca variação entre os tratamentos, porém a força de cisalhamento $\left(\mathrm{T} 1=4,08 ; \mathrm{T} 2=2,86\right.$ e $\left.\mathrm{T} 3=2,35 \mathrm{kgf} / \mathrm{cm}^{2}\right)$ e as perdas por cocção $(\mathrm{T} 1=31,97$; $\mathrm{T} 2=35,70$ e $\mathrm{T} 3=31,91 \%$ ) da carne diferiram entre os tratamentos. Não se observou alta correlação entre a maioria das características avaliadas.

Palavras-chave: ovino, correlação, força de cisalhamento, $\mathrm{pH}$ da carne

\begin{abstract}
The physical quality of the Longissimus lumborum muscle of Santa Inês culled ewes slaughtered at different physiological stages was evaluated as well as the color of the subcutaneous fat of the same muscle, and the correlations between meat quality traits. Twenty-one ewes were randomly allotted in the following treatments: $T 1=$ ewes which remained in lactation for 60 days with their respective lambs and slaughtered one day after weaning of the lambs; $T 2=$ ewes which remained in lactation for 60 days with their respective lambs, followed by an additional period of 30 days without the lambs and slaughtered afterwards; and T3= non pregnant ewes. The colors of meat and subcutaneous fat were not influenced by the physiological stage of the animals at the moment of slaughtering, except meat luminosity $\left(T 1=41.13 ; T 2=37.56\right.$ and $\left.T 3=38.12 L^{*}\right)$. The physical quality of the meat presented little variation between treatments; however, shear force $(T 1=4.08 ; T 2=2.86$ and $T 3=2.35 \mathrm{kgf} / \mathrm{cm} 2)$ and losses by cooking $(T 1=31.97 ; T 2=35.70$ and $T 3=31.91 \%)$ were affected by treatments. No high correlation between most of the evaluated traits was observed.
\end{abstract}

Keywords: sheep, correlations, meat pH, shear force

\section{INTRODUÇÃO}

A ovinocultura destinada à produção de carne vem crescendo e adquirindo cada vez mais importância na economia do país, porém a maioria dos ovinocultores desconhece qual o melhor estágio fisiológico para comercializar ovelhas de descarte, visando obter boas características qualitativas da carcaça e da carne.

Recebido em 29 de julho de 2009

Aceito em 22 de março de 2010

E-mail: rafaelsbp@bio.feis.unesp.br 
A qualidade da carne pode ser avaliada pelo $\mathrm{pH}$, cor, capacidade de retenção de água, maciez e perdas por cocção, e pelos aspectos sanitários e nutricionais. Tais características podem evidenciar carnes de melhor ou pior qualidade, e os resultados podem ser utilizados para determinar o preço dos produtos com distintas qualidades e serem direcionados para diferentes tipos de mercado.

As características de qualidade da carne são importantes na comercialização do produto (Brondani et al., 2006). As pesquisas que avaliaram a qualidade da carne ovina investigaram diferenças entre sexos (Gularte et al., 2000; Siqueira et al., 2001), idades de abate (Dawson et al., 2002), raças (Hopkins e Fogarty, 1998; Siqueira et al., 2002), alimentação (Tonetto et al., 2004; Madruga et al., 2005) e sistemas de produção (Astiz, 2008) e, na maioria dos casos, foram realizadas com cordeiros. Quanto aos ovinos adultos, estudos dessa natureza são escassos na literatura consultada. Os produtos desses animais são considerados de baixa qualidade, e não foram encontrados estudos que sinalizassem o melhor destino dos cortes cárneos, qual o momento de descarte desses animais (estágio fisiológico) e como prepará-los (tipo de sistema de criação, alimentação e manejo) para o abate.

Os objetivos deste trabalho foram estudar a qualidade física do músculo Longissimus lumborum, proveniente de ovelhas de descarte da raça Santa Inês, abatidas em distintos estágio fisiológicos, a cor (valores de $\mathrm{L}^{*}, \mathrm{a}^{*} \mathrm{e} \mathrm{b}^{*}$ ) da gordura subcutânea do mesmo músculo e as possíveis correlações entre as variáveis estudadas da qualidade da carne.

\section{MATERIAL E MÉTODOS}

O experimento foi realizado em uma cabanha comercial, localizada no município de Jaguariúna, no estado de São Paulo.

No manejo pré-experimental, foram colocadas 48 ovelhas de descarte da raça Santa Inês, com condição corporal de aproximadamente 2,5 , em estação de monta, por um período de 60 dias, com um reprodutor da mesma raça. O motivo do descarte dos animais foi por não mais se enquadrarem nos critérios de seleção adotados, diagnosticados por meio da escrituração zootécnica. Quando uma ovelha era coberta, anotava-se a data do ocorrido. As cobrições foram acompanhadas mediante marcação realizada com tinta colocada no peito do carneiro.

Sessenta dias após o término da estação de monta, foi realizado o diagnóstico de gestação e separação de 21 ovelhas para o experimento sete animais por tratamento -, que apresentaram média de peso corporal de $43 \pm 2,87 \mathrm{~kg}$ e de idade de $72 \pm 11$ meses. As fêmeas foram distribuídas nos seguintes tratamentos: $\mathrm{T} 1=$ ovelhas que permaneceram por 60 dias em lactação com seus respectivos cordeiros e abatidas um dia após o desmame das crias; $\mathrm{T} 2=$ ovelhas que permaneceram por 60 dias em lactação com seus respectivos cordeiros e mais um período aproximado de 30 dias sem as crias, no intuito de recuperar o peso corporal perdido durante $o$ período de amamentação, e posteriormente abatidas; e T3= ovelhas não paridas no ano e que permaneceram por 60 dias junto às ovelhas do T1 e T2 até o abate. O delineamento experimental utilizado foi o inteiramente ao acaso, com três tratamentos e sete repetições.

As ovelhas permaneceram em regime de confinamento em uma instalação coberta com área de solário e alimentação e água à vontade durante todo o período do experimento. Foram abatidas no mesmo dia, portanto as fêmeas do T2 pariram um mês antes das do T1, e todas tiveram partos simples.

A dieta fornecida apresentou relação volumoso:concentrado de 70:30. O volumoso foi o feno de Tifton - 85, e o concentrado constituído por $69,6 \%$ de grão de milho moído, 24,8\% de farelo de algodão, 0,8\% de calcário calcítico, $0,8 \%$ de fosfato bicálcico e $4 \%$ de suplemento vitamínico e mineral. Receberam duas refeições diárias, às $7 \mathrm{~h}$ e às $16 \mathrm{~h}$, em cocho de madeira que permitiu o acesso de todos os animais ao mesmo tempo. Os cordeiros tiveram acesso ao comedouro seletivo, onde foi oferecido concentrado à vontade, no intuito de minimizar o desgaste promovido pelo período de lactação das ovelhas. Todos os animais tiveram acesso ao sal comum, fornecido em cocho.

No manejo pré-abate, as ovelhas, em jejum de $16 \mathrm{~h}$ de dieta sólida e insensibilizadas com eletronarcose, foram submetidas à sangria pela 
secção das veias jugulares e artérias carótidas. Após a retirada da pele e das extremidades dos membros e da cabeça e evisceração, as carcaças foram transferidas para câmara frigorífica a $5^{\circ} \mathrm{C}$ por 24 horas. Ao final desse período, as carcaças foram divididas longitudinalmente em duas partes, retirando-se da meia carcaça direita o corte do lombo e deste corte, o músculo Longissimus lumborum, desprovido da gordura subcutânea. O músculo e a gordura subcutânea foram transferidos para uma geladeira, onde permaneceram por aproximadamente 24 horas, à temperatura de $6^{\circ} \mathrm{C}$, após o que se realizaram as análises de qualidade física da carne, em triplicata, e da cor da gordura subcutânea, também em triplicata.

A medida de $\mathrm{pH}$ foi determinada por meio de um peagômetro digital portátil, com introdução do eletrodo diretamente no músculo. Para determinar a cor da carne e da gordura subcutânea, foi utilizado colorímetro Minolta Chrome Meter CR-300, operando no sistema CIE (L* luminosidade, a* intensidade de vermelho, $b^{*}$ intensidade de amarelo), calibrado para um padrão branco em ladrilho. A capacidade de retenção de água foi calculada utilizando-se a metodologia descrita por Hamm (1960).

Para a realização das perdas por cocção, foi utilizado um forno industrial a gás pré-aquecido à temperatura de $170^{\circ} \mathrm{C}$. Amostras de carnes cruas foram pesadas e colocadas em bandejas com grelhas de ferro e novamente pesadas. Em seguida, foram transferidas para o forno, onde permaneceram até a temperatura interna do centro da amostra atingir $75^{\circ} \mathrm{C}$. Após serem resfriados (temperatura ambiente), foram novamente pesadas para o cálculo da porcentagem de perdas durante o cozimento. A temperatura interna da carne foi determinada com termômetro digital portátil tipo espeto.

As perdas por gotejamento foram calculadas pesando-se a bandeja com grelha de ferro antes do preparo da carne e após a cocção. As perdas por evaporação foram calculadas subtraindo-se da perda por cocção a perda por gotejamento. As perdas por gotejamento, por evaporação ou por cocção foram determinadas em percentagem. Para a força de cisalhamento, foram utilizadas as mesmas amostras da análise de perdas de água por cocção, as quais, após serem pesadas, foram cortadas em cilindros e submetidas ao corte no sentido transversal das fibras musculares, por meio de lâmina Warner-Bratzer acoplada ao aparelho Texture Analyser. Esses valores foram expressos em $\mathrm{kgf} / \mathrm{cm}^{2}$.

Para comparações de médias dos tratamentos, utilizou-se teste Tukey a 5\% de probabilidade e, para as análises de variância, o SAS/1996. Para determinar o coeficiente de correlação entre as variáveis estudadas nesta pesquisa, foi utilizada a correlação de Pearson, segundo o procedimento CORR do programa SAS/1996.

\section{RESULTADOS E DISCUSSÃO}

Os valores referentes à cor do músculo Longissimus lumborum e da gordura subcutânea são apresentados na Tab. 1. A luminosidade da carne diferiu entre os tratamentos experimentais $(\mathrm{P}<0,05)$, com maior valor para o $\mathrm{T} 1 \mathrm{em}$ relação ao T2; o valor de T3 foi próximo aos dos demais tratamentos. Os valores de $\mathrm{L}^{*}$ da carne estão próximos aos teores obtidos por Madruga et al. (2005), ao avaliarem a qualidade da carne de cordeiros da raça Santa Inês, e também aos obtidos por Rota et al. (2006), ao avaliarem a luminosidade da carne de ovinos abatidos com diferentes idades (120, 210 e 310 dias de vida).

Tabela 1. Valores médios da cor do músculo Longissimus lumborum e da gordura subcutânea, provenientes de ovelhas de descarte abatidas em distintos estágio fisiológicos

\begin{tabular}{lcccc}
\hline Variável & T1 & T2 & T3 & CV (\%) \\
\hline Cor da carne & & & & \\
$\mathrm{L}^{*}$ & $41,13 \mathrm{a}$ & $37,56 \mathrm{~b}$ & $38,12 \mathrm{ab}$ & 6,26 \\
$\mathrm{a}^{*}$ & 17,92 & 18,18 & 18,79 & 8,09 \\
$\mathrm{~b}^{*}$ & 3,94 & 3,56 & 3,55 & 14,91 \\
Cor da gordura & & & & \\
$\mathrm{L}^{*}$ & 68,15 & 69,26 & 70,50 & 5,19 \\
$\mathrm{a}^{*}$ & 6,27 & 7,15 & 6,27 & 15,92 \\
$\mathrm{~b}^{*}$ & 7,76 & 8,30 & 7,01 & 15,51 \\
\hline
\end{tabular}


T1: ovelhas abatidas um dia após o desmame das crias; T2: ovelhas abatidas 30 dias após o desmame das crias; T3: ovelhas não paridas.

Médias seguidas por letras distintas na linha diferem entre si pelo teste Tukey a $5 \%$ de probabilidade. L*= luminosidade; $a^{*}=$ intensidade de vermelho; $b^{*}=$ intensidade de amarelo. CV: coeficiente de variação.

De acordo com Sañudo et al. (1997), o teor de vermelho está relacionado com o conteúdo de mioglobina no músculo, e quanto maior for o seu valor, mais vermelha será a carne. Os valores de vermelho e amarelo obtidos não diferiram entre si $(\mathrm{P}>0,05)$, com valores médios de 18,29 e 3,68, respectivamente. Estes resultados são semelhantes aos obtidos por Pinheiro (2006), ao estudar a cor da carne de ovelhas e capões adultos de descarte. De acordo com esse autor, a carne oriunda de ovinos adultos é mais vermelha em relação à carne de cordeiros. Segundo Lawrie (2005), o aumento da concentração de mioglobina ocorre com a maturidade do animal.

A cor da gordura subcutânea do músculo Longissimus lumborum foi similar entre os tratamentos (Tab. 1). As xantofilas e os carotenos são os principais pigmentos responsáveis pela cor do tecido adiposo (Kirton et al., 1975). Segundo Forrest (1981), os ovinos não acumulam grandes quantidades desses pigmentos e, consequentemente, os depósitos adiposos deles apresentam coloração mais branca na maioria dos casos. A cor da gordura pode influenciar a preferência do consumidor no momento da compra da carne ovina, portanto são necessários estudos que avaliem a qualidade da gordura, além de estudos que determinem só a qualidade da carne desprovida de tecidos adiposos.

$\mathrm{Na}$ Tab. 2, constam os valores referentes à qualidade física da carne. $\mathrm{O}$ pH e a capacidade de retenção de água da carne foram similares $(\mathrm{P}>0,05)$ entre os tratamentos, com valores médios de 5,50 e 60,0\%, respectivamente. Os resultados obtidos neste estudo para a capacidade de retenção de água da carne são mais altos que os obtidos por Pinheiro (2006), que utilizou a mesma metodologia para determinar esta análise, ao avaliar diferentes músculos da carcaça, proveniente de distintas categorias de ovinos. Carnes com maior capacidade de retenção de água apresentam menores perdas de nutrientes por exsudato e normalmente são mais saborosas.

Ovelhas abatidas um dia após o desmame dos seus cordeiros apresentam carne com maior força de cisalhamento (Tab. 2) em relação à das ovelhas dos demais tratamentos, que não diferiram entre si $(\mathrm{P}>0,05)$, com valor médio de $2,60 \mathrm{kgf} / \mathrm{cm}^{2}$. Sañudo (2002) relatou que valores crescentes ou decrescentes para força de cisalhamento da carne ovina podem existir em função de interações entre diferentes taxas de deposição de colágeno e de gordura entremeada no músculo. A maciez da carne é uma das principais características de qualidade desejada pelo consumidor, portanto carnes mais tenras são normalmente mais valorizadas. As ovelhas de T2 e T3 apresentaram maciez do músculo Longissimus lumborum próximo ao valor obtido por Yamamoto (2006), no mesmo músculo estudado, ao utilizar a mesma metodologia de análise, porém obtidos na carne de cordeiros terminados em confinamento.

Tabela 2. Valores médios da qualidade do músculo Longissimus lumborum de ovelhas de descarte abatidas em diferentes estágio fisiológicos

\begin{tabular}{lcccc}
\hline Variável & $\mathrm{T} 1$ & $\mathrm{~T} 2$ & $\mathrm{~T} 3$ & $\mathrm{CV}(\%)$ \\
\hline $\mathrm{pH}^{1}$ & 5,54 & 5,51 & 5,48 & 1,37 \\
Capacidade de retenção de água $(\%)$ & 60,88 & 62,54 & 56,60 & 9,87 \\
Força de cisalhamento $\left(\mathrm{kgf} / \mathrm{cm}^{2}\right)$ & $4,08 \mathrm{a}$ & $2,86 \mathrm{~b}$ & $2,35 \mathrm{~b}$ & 9,30 \\
Perdas por cocção $(\%)$ & $31,97 \mathrm{~b}$ & $35,70 \mathrm{a}$ & $31,91 \mathrm{~b}$ & 8,64 \\
Perda por gotejamento $(\%)$ & 2,18 & 2,27 & 1,84 & 15,33 \\
Perda por evaporação $(\%)$ & 29,80 & 33,42 & 30,07 & 10,06 \\
\hline
\end{tabular}

T1: ovelhas abatidas um dia após o desmame das crias; T2: ovelhas abatidas 30 dias após o desmame das crias; T3: ovelhas não paridas.

Médias seguidas por letras distintas na linha diferem entre si pelo teste Tukey a $5 \%$ de probabilidade. ${ }^{1} \mathrm{pH}$ determinado na carne após 48 horas do abate do animal. CV: coeficiente de variação. 
Rota et al. (2004) obtiveram força de cisalhamento de $2,24 \mathrm{kgf} / \mathrm{cm}^{2}$ no músculo Longissimus dorsi de cordeiros abatidos com idade média de 180 dias de vida. Resultados semelhantes foram verificados por Rota et al. (2006), que avaliaram a qualidade do músculo Longissimus dorsi de ovinos abatidos em diferentes idades (120, 210 e 360 dias de vida), e constataram força de cisalhamento de 1,97, 2,34 e $2,52 \mathrm{kgf} / \mathrm{cm}^{2}$, respectivamente. Os resultados de força de cisalhamento da carne obtidos pelos autores citados são próximos aos obtidos no músculo Longissimus lumborum das ovelhas de descarte deste estudo para os T2 e T3. Portanto, o estágio fisiológico em que as ovelhas foram abatidas (T2 e T3) resultou em boa qualidade da carne, quanto ao atributo maciez, pois foram semelhantes aos resultados já obtidos por Rota et al. (2006) em ovinos jovens.

O tecido conectivo que confere resistência ao músculo e aos tendões é constituído principalmente pelo colágeno. Segundo Cranwell et al. (1996), o colágeno total é relativamente constante nos animais nas diferentes idades, mas, com o aumento da idade, as ligações tornam-se mais resistentes e estáveis, conferindo à carne maior resistência ao corte. Portanto, quando ocorre acréscimo de proteína, como nos animais em ganho compensatório, é necessária a formação adicional de colágeno, que é mais solúvel, resultando em carne mais macia. Cranwell et al. (1996) observaram que vacas de descarte, confinadas por 28 ou 56 dias, apresentaram maior quantidade de colágeno solúvel e carne mais macia que vacas não confinadas. Certamente, no músculo Longissimus lumborum das ovelhas de T2 e T3 ocorreu a formação de colágeno solúvel.

Os valores de perdas por cocção foram diferentes $(\mathrm{P}<0,05)$ entre os tratamentos (Tab. 2), com maiores perdas para a carne dos animais do T2 em relação aos de $\mathrm{T} 1$ e $\mathrm{T} 3$, que não diferiram entre si $(\mathrm{P}>0,05)$, com valor médio de $31,90 \%$. Durante a cocção da carne, ocorrem perdas quantitativas e qualitativas, e são desejáveis menores perdas durante o preparo da carne. Quanto às perdas por gotejamento e evaporação da carne durante sua cocção, elas não diferiram $(\mathrm{P}>0,05)$ entre os tratamentos.

O coeficiente de correlação de Pearson entre as avaliações de qualidade da carne de encontramse na Tab. 3 .

Tabela 3. Coeficiente de correlação de Pearson (r) e probabilidade (P) entre as características de qualidade da carne de ovelhas de descarte da raça Santa Inês medidas no músculo Longissimus lumborum

\begin{tabular}{|c|c|c|c|c|c|c|c|c|c|}
\hline Variável & & $\mathrm{pH}^{1}$ & $\mathrm{~L}^{*}$ & $a^{*}$ & $\mathrm{~b}^{*}$ & CRA & $\mathrm{FC}$ & PCC & PPG \\
\hline \multirow[t]{2}{*}{$\mathrm{L}^{*}$} & $\mathrm{P}$ & 0,12 & & & & & & & \\
\hline & $\mathrm{r}$ & $-0,33$ & & & & & & & \\
\hline \multirow[t]{2}{*}{$a^{*}$} & $\mathrm{P}$ & 0,01 & 0,55 & & & & & & \\
\hline & $\mathrm{r}$ & $-0,59$ & 0,13 & & & & & & \\
\hline \multirow[t]{2}{*}{$b^{*}$} & $\mathrm{P}$ & 0,16 & 0,01 & 0,03 & & & & & \\
\hline & $\mathrm{r}$ & $-0,32$ & 0,55 & 0,48 & & & & & \\
\hline \multirow[t]{2}{*}{ CRA } & $\mathrm{P}$ & 0,50 & 0,32 & 0,40 & 0,59 & & & & \\
\hline & $\mathrm{r}$ & 0,15 & $-0,23$ & $-0,19$ & $-0,12$ & & & & \\
\hline \multirow[t]{2}{*}{$\mathrm{FC}$} & $\mathrm{P}$ & 0,07 & 0,26 & 0,07 & 0,97 & 0,17 & & & \\
\hline & $\mathrm{r}$ & 0,40 & 0,25 & $-0,39$ & 0,07 & 0,31 & & & \\
\hline \multirow[t]{2}{*}{ PCC } & $\mathrm{P}$ & 0,39 & 0,79 & 0,57 & 0,78 & 0,68 & 0,85 & & \\
\hline & $\mathrm{r}$ & $-0,20$ & $-0,06$ & 0,13 & 0,06 & 0,09 & $-0,04$ & & \\
\hline \multirow[t]{2}{*}{ PPG } & $\mathrm{P}$ & 0,63 & 0,81 & 0,32 & 0,65 & 0,04 & 0,82 & 0,88 & \\
\hline & $\mathrm{r}$ & $-0,11$ & 0,05 & 0,22 & 0,10 & 0,44 & 0,05 & 0,03 & \\
\hline \multirow[t]{2}{*}{ PPE } & $\mathrm{P}$ & 0,50 & 0,74 & 0,82 & 0,90 & 0,81 & 0,80 & 0,01 & 0,19 \\
\hline & $\mathrm{r}$ & $-0,15$ & $-0,07$ & 0,05 & 0,03 & $-0,05$ & $-0,06$ & 0,94 & $-0,29$ \\
\hline
\end{tabular}

T1: ovelhas abatidas um dia após o desmame das crias; T2: ovelhas abatidas 30 dias após o desmame das crias; T3: ovelhas não paridas.

Médias seguidas por letras distintas na linha diferem entre si pelo teste Tukey a $5 \%$ de probabilidade. ${ }^{1} \mathrm{pH}$ determinado na carne após 48 horas do abate do animal. CRA: Capacidade de retenção de água; FC: força de cisalhamento; PCC: perdas por cocção; PPG: perda por gotejamento; PPE: perda por evaporação. L*= luminosidade; $\mathrm{a}^{*}=$ intensidade de vermelho; $\mathrm{b}^{*}=$ intensidade de amarelo. 
Não se observou $(P>0,05)$ alta correlação entre a maioria das características de qualidade da carne estudadas (Tab. 3). A correlação negativa $(\mathrm{P}<0,01)$ entre o $\mathrm{pH}$ e o teor de vermelho da carne indica valores menores de $\mathrm{a}^{*}$ e maiores de pH ou vice-versa. De acordo com Melo (1985), citado por Marques (2006), a cor da carne não é determinada apenas pelo teor de mioglobina e pela forma molecular na qual se encontra, mas também pelo $\mathrm{pH}$.

Observou-se correlação significativa entre o teor de amarelo e os valores de $\mathrm{L}^{*}$ e a*. Entre as perdas por cocção e perdas por evaporação, observou-se alta correlação (Tab. 3). Assim, quanto maior for a perda por evaporação maior será a perda por cocção. Perda por gotejamento e capacidade de retenção de água da carne apresentaram baixa correlação.

\section{CONCLUSÕES}

O estágio fisiológico de ovelhas de descarte da raça Santa Inês influencia a maciez, a luminosidade e as perdas por cocção do músculo Longissimus lumborum. Valores mais altos de $\mathrm{pH}$ indicam teores mais baixos de vermelho na carne, ou vice-versa. Quanto maior o teor de amarelo maiores serão os valores de luminosidade e de intensidade de vermelho da carne ovina.

\section{REFERÊNCIAS BIBLIOGRÁFICAS}

ASTIZ, C.S. Qualidade da carcaça e da carne ovina e caprina em face ao desenvolvimento da percepção do consumidor. Rev. Bras. Zootec., v.37, supl. esp., p.143-160, 2008.

BRONDANI, I.L.; SAMPAIO, A.A.M.; RESTLE, J. et al. Composição física da carcaça e aspectos qualitativos da carne de bovinos de diferentes raças alimentados com diferentes níveis de energia. Rev. Bras. Zootec., v.35, p.2034-2042, 2006.

CRANWELL, C.D.; UNRUH, J.A.; BRETHOUR, J.R. et al. Influence of steroid implants and concentrate feeding on carcass an Longissimus muscle sensory and collagen characteristics of cull beef cows. J. Anim. Sci., v.74, p.1777-1783, 1996.
DAWSON, L. E. R.; CARSON, A. F.; MOSS, B. W. Effects of crossbred ewe genotype and ram genotype on lamb meat quality from the lowland sheep flock. J. Anim. Sci., v.13, p.195-204, 2002.

FORREST, R.J. Effect of high concentrate feeding on the carcass quality and fat coloration of grass reared steers. Can. J. Anim. Sci., v.61, p.575-580, 1981.

GULARTE, M.A.; TREPTOW, R.O.; POUEY, J.L.F. et al. Idade e sexo na maciez da carne de ovinos da raça Corriedale. Cienc. Rural, v.30, p.485-488, 2000.

HAMM, R. Biochimistry of meat hydratation. Adv. Food Res., v.10, p.335-443, 1960.

HOPKINS, D.L.; FOGARTY, N.M. Diverse lamb genotypes. 2. Meat $\mathrm{pH}$, colour and tenderness. Meat Sci., v.49, p.477-488, 1998.

KIRTON, A.H.; CRANE, B.; PATERSON, D.J. et al. Yellow fat in lambs caused by carotenoid pigmentation. N. Z. J. Agric. Res., v.18, p.267$272,1975$.

LAWRIE, R.A. Ciência da carne. 6.ed. Porto Alegre: Artmed, 2005. 384p.

MADRUGA, M.S.; SOUSA, W.H.; ROSALES, M.D. et al. Qualidade da carne de cordeiros Santa Inês terminados com diferentes dietas. Rev. Bras. Zootec., v.34, p.309-315, 2005.

MARQUES, A.V.M.S. Características quantiqualitativas da carcaça e da carne de cordeiros Santa Inês alimentados com diferentes níveis de feno de flor-de-seda (Calotropis procera $\mathrm{SW}$ ) em substituição ao feno de sorgo forrageiro (Sorghum bicolor L). 2006. 77f. Tese (Doutorado) - Universidade Federal da Paraíba, João Pessoa.

PINHEIRO, R.S.B. Aspectos quantitativos da carcaça e qualitativos da carne de ovinos de diferentes categorias. 2006. 105f. Dissertação (Mestrado) - Faculdade de Ciências Agrárias e Veterinárias, Universidade Estadual Paulista, Jaboticabal, SP.

ROTA, E.L.; OSÓRIO, M.T.M.; OSÓRIO, J.C.S. et al. Influência da castração e da idade de abate sobre as características subjetivas e instrumentais da carne de cordeiros Corriedale. Rev. Bras. Zootec., v.35, p.2397-2405, 2006.

ROTA, E.L.; OSÓRIO, M.T.M.; OSÓRIO, J.C.S. et al. Efeitos do cruzamento de carneiros 
da raça Texel com ovelhas Corriedale e Ideal sobre a qualidade da carne. Rev. Bras. Agroc., v.10, p.487-491, 2004.

SAÑUDO, C. Factors affecting carcass and meat quality in lambs. In: REUNIÃO ANUAL DA SOCIEDADE BRASILEIRA DE ZOOTECNIA, 39., 2002, Recife. Anais... Recife: SBZ, 2002. p.434-455.

SAÑUDO, C.; CAMPO, M.M.; SIERRA, I. et al. Breed effect on carcass and meat quality of suckling lambs. Meat Sci., v.46, p.357-365, 1997.

SIQUEIRA, E. R.; SIMÕES, C.D.; FERNANDES, S. Efeito do sexo e do peso ao abate sobre a produção de carne de cordeiros. I. velocidade de crescimento, caracteres quantitativos da carcaça, pH da carne e resultados econômicos. Rev. Bras. Zootec., v.30, p.844-848, 2001.
SIQUEIRA, E.R.; ROCA, R.O.; FERNANDES, $\mathrm{S}$. et al. Características sensoriais da carne de cordeiros das raças Hampshire Down, Santa Inês e Mestiços Bergamácia x Corriedale abatidos com quatro distintos pesos. Rev. Bras. Zootec., v.31, p.1269-1272, 2002.

TONETTO, C.J.; PIRES, C.C.; MÜLLER, L. et al. Rendimentos de cortes da carcaça, características da carne e componentes do peso vivo em cordeiros terminados em três sistemas de alimentação. Rev. Bras. Zootec., v.33, p.234241, 2004.

YAMAMOTO, S.M. Desempenho e características de carcaça de cordeiros terminados em confinamento com dietas contendo silagem de peixe. 2006. 95f. Tese (Doutorado) - Faculdade de Ciências Agrárias e Veterinárias, Universidade Estadual Paulista, Jaboticabal, SP. 